\title{
Das Hüftsonographie-Screening - einige Gedanken eines Gesundheitsökonomen
}

\author{
W. Oggier
}

Das Hüftsonographie-Screening ist Ende März 2004 von der Streichung aus dem Leistungskatalog der sozialen Krankenversicherung bedroht. Der Autor argumentiert aus drei unterschiedlichen gesundheitsökonomischen Sichtweisen und kommt dabei zum Schluss, dass eine Streichung auf dem heutigen Wissensstand gesundheitsökonomisch nicht zu rechtfertigen sein dürfte. Verbesserungswürdig hält er die Anforderungen an die Wirtschaftlichkeit. Er regt ausserdem an, die anfallenden Kosten nicht nur aus der Warte der Kranken-, sondern auch der anderen Sozialversicherungen, insbesondere der Invalidenversicherung, zu betrachten.

1 Ahrens D. Technologiebewertung und Public Health. Bern: Verlag Hans Huber; 2002.

2 Vgl. dazu beispielsweise Schöffski O Gendiagnostik: Versicherung und Gesundheitswesen - eine Analyse aus ökonomischer Sicht. Karlsruhe: Verlag Versicherungswirtschaft; 2000, oder Greenhalph T. Einführung in die Evidence-based Medicine. Bern: Verlag Hans

Huber; 2000.

\section{Einleitung}

Das Hüftsonographie-Screening könnte Ende März 2004 vom Leistungskatalog der obligatorischen Krankenversicherung gestrichen werden. Im folgenden soll aus drei unterschiedlichen Blickwinkeln anhand einiger gesundheitsökonomischer Überlegungen der Frage nachgegangen werden, ob eine solche Streichung Sinn macht. In einem ersten Schritt werden dabei Kriterien herangezogen, welche generell für Screeningverfahren postuliert werden. Danach sollen ausgehend vom Magischen Fünfeck gesundheitspolitischer Zielsetzungen bzw. von den Grundsätzen des eidgenössischen Krankenversicherungsgesetzes (KVG) weitere Erörterungen folgen.

Für den Fall des Hüftsonographie-Screenings ist die schweizerische Datenlage aus gesundheitsökonomischer Sicht als schlecht $\mathrm{zu}$ bezeichnen. Dies ist jedoch keine hüftsonographische Eigenheit, sondern widerspiegelt die generell schlechte Datenlage im schweizerischen Gesundheitswesen. Die Gesundheitsökonomie kommt daher nicht umhin, mit Annahmen zu arbeiten bzw. auf ausländische Erfahrungen abzustützen, wenn das Hüftsonographie-Screening näher analysiert werden soll.

\section{Screeningkriterien}

Allgemeine Kriterien für die Einführung von Screeningprogrammen wurden 1968 von der UN-Weltgesundheitsorganisation WHO formu- liert und sind aktualisiert worden (z.B. www. cancerscreening.nhs.uk/). Im wesentlichen handelt es sich dabei um folgende Kriterien [1]:

- die zu entdeckende Krankheit muss ein bedeutendes Gesundheitsproblem darstellen;

- eine allgemein akzeptierte Behandlung sollte verfügbar sein;

- es sollten Einrichtungen für die Diagnose und Behandlung verfügbar sein;

- es sollte einen erkennbar latenten oder früh symptomatischen Zustand geben;

- es sollte ein passender Test oder eine passende Untersuchung zur Verfügung stehen;

- der Test sollte in der Bevölkerung akzeptiert sein;

- die Krankheitsgeschichte einschliesslich des Übergangs von der latenten zur manifesten Phase sollte adäquat verstanden sein;

- es sollte Einigkeit darüber bestehen, wer als Patient behandelt wird;

- die Kosten der Entdeckung und Behandlung eines Falls sollten im Verhältnis zu den Gesamtmöglichkeiten des Gesundheitssystems ausbalanciert sein;

- das Screeningprogramm sollte einen kontinuierlichen Prozess und nicht ein einmaliges Projekt darstellen.

In Ergänzung dazu sind in der Zwischenzeit weitere Kriterien entwickelt worden. Dazu gehören beispielsweise [2]:

- die Teilnahme am Screeningprogramm soll freiwillig und aufgrund informierter Zustimmung erfolgen;

- Information der getesteten Personen über die positiven und negativen Testergebnisse;

- nicht-direktive Beratung vor dem Test und (bei positivem Ergebnis) nach dem Test;

- Gewährleistung der Datensicherheit und der Privatsphäre;

- gleicher Zugang zu den Testmöglichkeiten für alle Betroffenen;

- die Teilnahmerate der (Teil-)Populationen sollte möglichst hoch sein;

- Gefährdungen, die von der Früherkennung ausgehen, sollten möglichst klein sein. 
Wie steht es nun um diese Kriterien beim Hüftsonographie-Screening?

Im Gegensatz zu anderen Screeningmassnahmen können die formulierten Kriterien auf dem heutigen Wissensstand weitgehend erfüllt werden. Im Rahmen dieses Artikels soll angesichts der zur Verfügung stehenden Zeilen nur auf einige wenige dieser Kriterien eingegangen werden. Die Häufigkeit der Neuerkrankungen (Inzidenz) der angeborenen Hüftdysplasie liegt gemäss neueren deutschen Daten bei rund 1 pro 1000 Lebendgeburten. Im Vergleich zu anderen Bereichen, wo ein Screening diskutiert wird wie beispielsweise beim Zervixkarzinom (weltweite Inzidenzraten zwischen 5 und 48 Frauen pro 100000), fällt die Inzidenz der Hüftdysplasie höher aus. Mit der Methode nach Graf ist auch eine allgemein akzeptierte und sehr gute Screeningmethode verfügbar. Im Gegensatz etwa zum MammographieScreening kann beim heutigen Wissensstand auch davon ausgegangen werden, dass keine Gefährdungen wie Strahlenbelastungen von der Früherkennung ausgehen. Ein falsch positives Ergebnis dürfte auch kaum nachhaltige Effekte auf die psychische Befindlichkeit zeitigen. Probleme bereiten dürfte in erster Linie die Information der getesteten Personen über die positiven und negativen Testergebnisse. Dieses Problem kommt in der ganzen Pädiatrie vor und ist nicht screeningspezifisch, weil das Kleinkind in der Regel die ärztlichen Informationen nicht angemessen zu gewichten vermag bzw. gar nicht versteht. Geht man aber von der Annahme aus, dass die Eltern in Stellvertretung für das Kind korrekt informiert werden, dürfte auch dieses Kriterium erfüllt werden können.

\section{Magisches Fünfeck gesundheits- politischer Zielsetzungen}

Bereits während der achtziger Jahre haben Ökonominnen und Ökonomen in Anlehnung an das Fünfeck der wirtschaftspolitischen Zielsetzungen ein solches für die gesundheitspolitischen Zielsetzungen entwickelt [3]. Die einzelnen Ziele können bezogen auf die Hüftsonographie auf folgende Art umschrieben werden:

- Chancengleichheit: Aus Erfahrungen mit anderen präventiven Massnahmen kann davon ausgegangen werden, dass der chancengleiche Zugang zu den Angeboten des Gesundheitswesens beeinträchtigt werden dürfte, wenn das Hüfsonographie-Screening von Eltern aus sozial schwächeren Schichten selbst bzw. über eine Zusatzversicherung bezahlt werden müsste. Es scheint mindestens a priori einiges dafür zu sprechen, dass Säuglinge davon betroffen sein können. Denn die in den letzten Jahren entstandenen Armutsstudien haben für die Schweiz fast alle das grösste Armutsrisiko bei alleinerziehenden Müttern ausgemacht. Ähnliches lässt sich beispielsweise auch bei Schwangerschaftsvorsorgeuntersuchungen ausmachen, die von Ausländerinnen wesentlich weniger genutzt werden als von deutschen Frauen. Zimmermann führt dieses Ergebnis auf verschiedene Faktoren zurück (geringe Erfahrung mit solchen Massnahmen, erhöhtes Schamgefühl, unzureichende Sprachkenntnisse, prekäre sozioökonomische Lage) [4].

- Leistungsfähigkeit: Die Hüftsonographie ermöglicht bei einem grösseren Anteil der von einer Hüftdysplasie betroffenen Säuglinge eine frühzeitigere Diagnose und Behandlung als alle anderen alternativen Verfahren. In einer kürzlich publizierten Studie konnte dokumentiert werden, dass nach der Einführung des Hüftsonographie-Screenings in Deutschland ein Rückgang der operativen Korrekturen der Hüftdysplasien zu verzeichnen ist: heute kommt 1 Operation auf 4000 Lebendgeburten gegenüber einer Inzidenz von 1:1000 in den Jahren 1977 bis 1983 [5]. Im Gegensatz zu anderen Screeningprogrammen kann der Behandlungserfolg auch kaum systematisch überschätzt sein, weil der Therapiezeitpunkt nicht beliebig nach vorne verlegt werden kann. Das Kind muss ja zuerst einmal geboren sein.

- Bedarfsgerechtigkeit: Aus gesundheitsökonomischer Sicht wird oft befürchtet, dass die Leistungserbringer nach Anerkennung der Leistungspflicht für die Grundversicherung insbesondere bei einer Entschädigung auf der Basis einer Einzelleistungsvergütung versucht sein könnten, Strategien der Mengenausweitung zu betreiben. Dieser in der gesundheitsökonomischen Literatur gut dokumentierte Zusammenhang dürfte beim Hüftsonographie-Screening kaum auszumachen sein. Einerseits dürften die Ärztinnen und Ärzte die Anzahl Neugeborener selbst nicht signifikant beeinflussen können. Anderseits dürfte die Anzahl Nachkontrollen zwischen 15 und 20 Prozent der untersuchten Kinder liegen. Hinzu kommt, dass die Hüftsonographie bei Säuglingen tariflich gesehen zurzeit nicht besonders interessant sein dürfte, was in der Regel auf Mengenausweitungsstrategien eher dämpfend als fördernd wirkt. Dieser Effekt dürfte es zudem 
vor allem den mit dem Verfahren routinierten Pädiatern ermöglichen, kostendeckend zu arbeiten. Eine höhere Anzahl untersuchter Fälle dürfte wiederum die Qualität der ausgeführten Untersuchung verbessern.

- Wirtschaftlichkeit: Für die Schweiz liegt keine umfassende gesundheitsökonomische Evaluation vor. Die deutsche Studie von von Kries u.a. bringt allerdings zum Ausdruck, dass die Wirtschaftlichkeit des Verfahrens noch erhöht werden könnte. Der Idealzustand wäre gesundheitsökonomisch gesehen dann erreicht, wenn mit den aufgewendeten Mitteln alle Operationen bei entwicklungsbedingter Hüftdysplasie verhindert werden könnten. Neben anderen Gründen wie unauffälliger Ultraschallbefund scheint die Wirtschaftlichkeit aber durch die Häufigkeit der rechtzeitigen Untersuchungen, also bis zur sechsten Woche, und die Schulung von Ärzten in der Ultraschallüberprüfung weiter verbessert werden zu können. Aus gesundheitsökonomischer Sicht ergänzt werden kann, dass auch die Erfahrung des Arztes mit dem entsprechenden Verfahren mittel- bis langfristig einen entsprechenden Einfluss auf die Wirtschaftlichkeit der erbrachten Leistung haben dürfte.

- Finanzierbarkeit: Im Rahmen der obligatorischen Krankenversicherung geht es aber auch um die Beachtung der Finanzierbarkeit. Selbst wenn man unterstellen würde, dass das generelle Hüftsonographie-Screening doppelt so teuer zu stehen käme, wie die Screeningbefürworter aus Ärztekreisen unterstellen (Annahme: 300 statt 150 Franken, jeweils inkl. ärztliche Konsultation), ergäben sich bei einer 100\%-Screening-Rate für das Jahr 2000 (rund 78000 Neugeborene) Kosten im Umfang von 23,4 Millionen Franken pro Jahr. Ohne Einbezug des Nutzens aus den entgangenen Kosten durch ein Screeningprogramm entspricht dies - bezogen auf die gesamten durch die obligatorische Krankenversicherung bezahlten Leistungen gemäss den provisorischen Ergebnissen der Statistik über die Krankenversicherung im Jahr 2002 von 14,6 Milliarden Franken - einem Anteil von 0,16 Prozent der gesamten bezahlten Leistungen. Bezogen auf das Prämiensoll aus dem Jahr 2002 entspricht dieser Betrag einem Anteil von 0,15 Prozent. Diese Daten dürften auch aus dem Grund als zu hoch erscheinen, als die Anzahl Geburten im Jahr 2002 bei rund 72000, im Jahr 2003 bei rund 71500 lag.

\section{KVG-Kriterien}

Das KVG sieht in Artikel 32 vor, dass die obligatorische Krankenversicherung Leistungen nur zu bezahlen hat, wenn diese wirksam, zweckmässig und wirtschaftlich sind. Die Wirksamkeit muss nach wissenschaftlichen Methoden nachgewiesen sein. Die Wirksamkeit, die Zweckmässigkeit und die Wirtschaftlichkeit der Leistungen werden periodisch überprüft.

Was wirksam, zweckmässig und wirtschaftlich heisst, darüber streiten sich die Akteure im schweizerischen Gesundheitswesen. Aus der Sicht der Krankenversicherer lassen sich die drei Begriffe auf folgende Art beschreiben [6]:

- Wirksam heisst: Der erwünschte diagnostische oder therapeutische Effekt kann mit der Leistung erreicht werden.

- Zweckmässig heisst: Der erzielte Nutzen überwiegt dabei gegenüber unerwünschten Nebenwirkungen bzw. das Verhältnis Nutzen/Nebenwirkungen ist mindestens gleich gut oder besser als bei anderen in Frage kommenden Leistungen.

- Wirtschaftlich heisst: Der angestrebte Erfolg wird zu möglichst günstigen Kosten erreicht. Und es heisst auch: Eine neue Leistung darf nur dann mehr kosten als eine bereits anerkannte, wenn sie entsprechend mehr Nutzen bringt.

Der Nachweis der Wirksamkeit des Hüftsonographie-Screenings ist durch die Arbeiten der Arbeitsgruppe von Rüdiger von Kries von der Ludwigs-Maximilians-Universität in München erbracht worden. Sie stellt für die schweizerische Diskussion ein gewichtiges Argument dar, weil es sich um ein benachbartes Land handelt, mit dem auch in anderen gesundheitspolitischen Bereichen Vergleiche angestellt werden. Es handelt sich zudem um eine kürzlich publizierte Studie in einer der weltweit angesehensten medizinischen Fachzeitschriften. Und die Studie vergleicht die Zustände vor und nach der Einführung des Hüftsonographie-Screenings.

Die Zweckmässigkeit kann zweifellos bejaht werden, insbesondere im Vergleich zu den nicht monetarisierbaren, aber bei diesem Krankheitsbild im wahrsten Sinn des Wortes lebenslänglich möglichen Folgen. Betrachtet man die Zweckmässigkeit ausschliesslich aus dem Blickwinkel der direkt anfallenden Kosten, ist zwischen den Gesamtwirkungen und den Wirkungen auf die einzelnen Finanzierer zu unterscheiden. Es kann nicht ganz ausgeschlossen werden, dass eine Finanzierung des Hüftsonographie-Screenings 
durch die obligatorische Krankenversicherung den Krankenversicherer mehr kostet als die vermiedenen Behandlungskosten als Folge der rechtzeitigen Entdeckung durch das Screening. Entlastet werden dürfte dafür die Invalidenversicherung, welche für die entstehenden Geburtsgebrechen bis zum 20. Altersjahr aufzukommen hat. Der Umstand, dass der Finanzierer einer Leistung nicht mit dem Nutzniesser der Leistung zusammenhängt, ist im schweizerischen Gesundheitswesen nichts Ungewöhnliches, sondern ein systembedingter, gesundheitsökonomisch als schwerwiegend $\mathrm{zu}$ taxierender Fehler. Wegen dieser Systemverzerrung sollte bei der Beurteilung des Hüftsonographie-Screenings nicht ausschliesslich der Standpunkt der Krankenversicherung berücksichtigt werden, sondern auch jener der Invalidenversicherung. Die Annahme dürfte wohl nicht ganz abwegig erscheinen, dass die Diskussion über die Leistungspflicht des Hüftsonographie-Screenings kaum stattfinden würde, wenn die Unfallversicherung dafür aufkommen müsste. Denn diese müsste die Folgekosten insbesondere auch bei den Taggeldern finanzieren und verfügt daher in der Regel über eine langfristigere Optik.

Die Wirtschaftlichkeit des HüftsonographieScreenings dürfte insbesondere von der Einhaltung qualitativer Anforderungen abhängig sein. Die folgende Aufzählung zeigt, dass Qualität und Wirtschaftlichkeit im Gesundheitswesen keine Gegensätze zu sein brauchen. Folgende Faktoren dürften für die Wirtschaftlichkeit von Bedeutung sein:

- Es ist wichtig, dass die entsprechenden Leistungen den qualitativen Anforderungen an die Ausführung vollumfänglich entsprechen und einer Qualitätssicherung unterzogen werden.

- Auf den bestehenden TARMED-Grundlagen kommt dem Umstand hohe Bedeutung zu, welche Positionen in welchem Umfang zu welchen Taxpunktwerten abgerechnet werden. Ausserdem besteht aus gesundheitsökonomischer Sicht in der Regel auch eine generelle Skepsis gegenüber der Einzelleistungsvergütung und eine gewisse Priorität zu pauschalen, im voraus vereinbarten Preisen.

- Ebenfalls von hoher Relevanz ist die Anzahl Nachkontrollen, welche notwendig werden. Diese dürften u.a. von den entsprechenden Qualitätsanforderungen und vom Zeitpunkt abhängen, an dem Hüftsonographien durchgeführt werden. Zu prüfen wäre ausserdem, ob nicht eine minimale Anzahl Fälle pro Jahr und zugelassenem Leistungserbringer erforderlich sind, um diese Leistung zulasten der sozialen Krankenversicherung abzurechnen.

- Berücksichtigt werden sollte zudem, dass besonders betroffene Gruppen bzw. Schichten Untersuchungen bei reinen «Komm-Strukturen» besonders wenig in Anspruch nehmen. Zu prüfen sind daher auch entsprechende Informationsmassnahmen für die betroffenen Bevölkerungsteile.

Die Forderungen zur Wirtschaftlichkeit beziehen sich aber nicht nur auf das HüftsonographieScreening, sondern sollten analog bei anderen Pflichtleistungen aus der sozialen Krankenversicherung vermehrt zur Geltung kommen. Sie dürften im gegenwärtigen Zeitpunkt jedoch keinesfalls rechtfertigen, dass das HüftsonographieScreening aus dem Leistungskatalog gestrichen wird. Denn die Rahmenbedingungen zur Einhaltung der Wirtschaftlichkeit könnten entsprechend formuliert werden. Allerdings wäre dann zu postulieren, dass die entsprechenden Anforderungen im Sinne der Rechtsgleichheit auch für die anderen KVG-Pflichtleistungen angewendet werden.

Hinzu kommt, dass sich das Beispiel des Hüftsonographie-Screenings im Vergleich zu anderen bisher vom sozialen Krankenversicherer übernommenen Pflichtleistungen angesichts der oben dargestellten wirtschaftlichen Bedeutung für die soziale Krankenversicherung, aber auch mit Blick auf die Unvermeidbarkeit der Hüftdysplasie durch den Säugling wohl kaum als Paradebeispiel zur Streichung von Leistungen aus dem Leistungskatalog eignet.

\section{Fazit}

Die dargelegten gesundheitsökonomischen Gründe dürften es auf dem heutigen Wissensstand nicht rechtfertigen, das HüftsonographieScreening aus dem Leistungskatalog der sozialen Krankenversicherung zu streichen. Verbesserungsmöglichkeiten ergeben sich bei der Wirtschaftlichkeit. Diese sind jedoch realisierbar, ohne das Hüftsonographie-Screening aus dem Leistungskatalog zu streichen. 